\title{
The Nanofocus Endstation of the MINAXS Beamline of PETRA III
}

\author{
C.A. Krywka ${ }^{a}$, R. Döhrmann ${ }^{b}$, S.V. Roth ${ }^{b}$, and M. Müller ${ }^{\mathrm{c}}$ \\ IEAP, Christian-Albrechts-Universität zu Kiel, Leibnizstraße 19, D-24098 Kiel, Germany \\ ${ }^{b}$ DESY, Notkestraße 85, D-22063 Hamburg, Germany \\ ${ }^{c}$ GKSS Forschungszentrum Geesthacht, Max-Planck-Straße 1, D-21502 Geesthacht, Germany
}

\begin{abstract}
The Micro- and Nanofocus X-ray Scattering Beamline (MINAXS) of the new $3^{\text {rd }}$ generation source PETRA III is equipped with two endstations, out of which the farthest from the high beta undulator source is designed to provide a high flux, monochromatic X-ray beam focused to a size in the order of $100 \mathrm{~nm} \times 100 \mathrm{~nm}$ routinely used for microdiffraction experiments (nanofocus endstation). This contribution presents an overview on the current status of the nanofocus endstation and outlines the to-be-used experimental setup.
\end{abstract}

Keywords: Nanodiffraction, X-ray focusing, SAXS, WAXS

PACS: 61.05.C-, 61.05.cf, 61.05.cp

\section{THE MINAXS BEAMLINE AT PETRA III}

The new $3^{\text {rd }}$ generation synchrotron radiation source PETRA III is currently in its final state of completion and first beamlines are planned to take up user operation by the end of 2009. Located on the site of DESY in Hamburg (Germany) the former PETRA storage ring was completely refurbished with the aim to create one of the most brilliant X-ray sources worldwide and a $280 \mathrm{~m}$ section of the $2.3 \mathrm{~km}$ circumference was replaced with an all new experimental hall containing 14 newly built beamlines [1].

The MIcro- and NAnofocus X-ray Scattering beamline (MINAXS) has an overall length of about $100 \mathrm{~m}$ and is equipped with two consecutive endstations [2]. The farther of these two is the nanofocus endstation, starting at about $97 \mathrm{~m}$ from the high beta undulator source, and is designed to deliver a high flux, monochromatic X-ray beam (energy range 8 to $23 \mathrm{keV}$ ) routinely focused to a size of about $100 \mathrm{~nm} \times 100 \mathrm{~nm}$ or smaller. The nanofocus endstation will primarily serve for nanodiffraction experiments, that is small- and wide-angle X-ray scattering (SAXS and WAXS, respectively). Due to the submicron focus diffraction these types of experiments can be performed with a superior spatial resolution and a flux sufficiently high to study both biological and synthetic materials. Commissioning is planned to take up in 2010.

\section{THE NANOFOCUS ENDSTATION AT MINAXS}

Shown in figure 1 is a $3 \mathrm{D}$ model of the experimental setup to be used in the nanofocus endstation. The entire setup is mounted on a heavy, yet movable on air-pads, two-segment granite table with a top face size of $2.5 \times 0.8 \mathrm{~m}^{2}$. The beam height above ground is $90 \mathrm{~cm}$ (42 cm above table), being a consequence of the large offset monochromator employed at the beamline, hence improving the apparatus' mechanical stability by its low center of mass.

Both, the alignment of the sample as well as that of the closely preceding optical element (that is a $2 \mathrm{D}$ waveguide or at a later stage a focusing mirror) are based on hexapods. The main advantage of this type of adjustment is a highly flexible integration of custom sample environments. The two hexapods each provide 3 linear and 3 rotational degrees of freedom with a freely definable pivot point and a positioning reproducibility of $300 \mathrm{~nm}$ and $100 \mathrm{~nm}$, respectively. 
Each of the hexapods is equipped with a top-mounted $\mathrm{XZ}$ piezo stage providing a positioning reproducibility of $<10 \mathrm{~nm}$ to match the targeted, superior spatial resolution of the experiment. The entire sample alignment hexapod can be moved out of the beam as far as $50 \mathrm{~cm}$ for a convenient sample mounting and exchange.

The sample position can be observed by means of two video microscopes with large focal distances. Together with a set of movable tilted mirrors the observation can be performed in any of the three orthogonal directions. Observation along the beam direction remains possible even during X-ray irradiation, that is without interfering with the incident beam. To facilitate the alignment of the sample into the X-ray beam a visible guiding laser beam can be coupled into the X-ray path at any time and a laser spot with a diameter of $\sim 100 \mu \mathrm{m}$ (FWHM) is then projected onto the sample, hence becoming clearly visible in the in-beam observation direction. For the process of sample alignment as well as for the actual experiment a fluorescence detector can be used as it is also mounted onto the sample alignment hexapod and is directed onto the sample position.

The X-ray beam focal spot can be monitored by using one of the two installed X-ray beam cameras. These primary beam resistant CCD cameras can be moved into the beam if required and provide a resolution of $\sim 10 \mu \mathrm{m}$ and $\sim 1 \mu \mathrm{m}$, with a field of view of $\sim(10 \mathrm{~mm})^{2}$ and $\sim(0.5 \mathrm{~mm})^{2}$, respectively. Changes in the size and geometry of the beam spot can therefore be easily monitored and the process of creating and optimizing the focal spot will be facilitated.

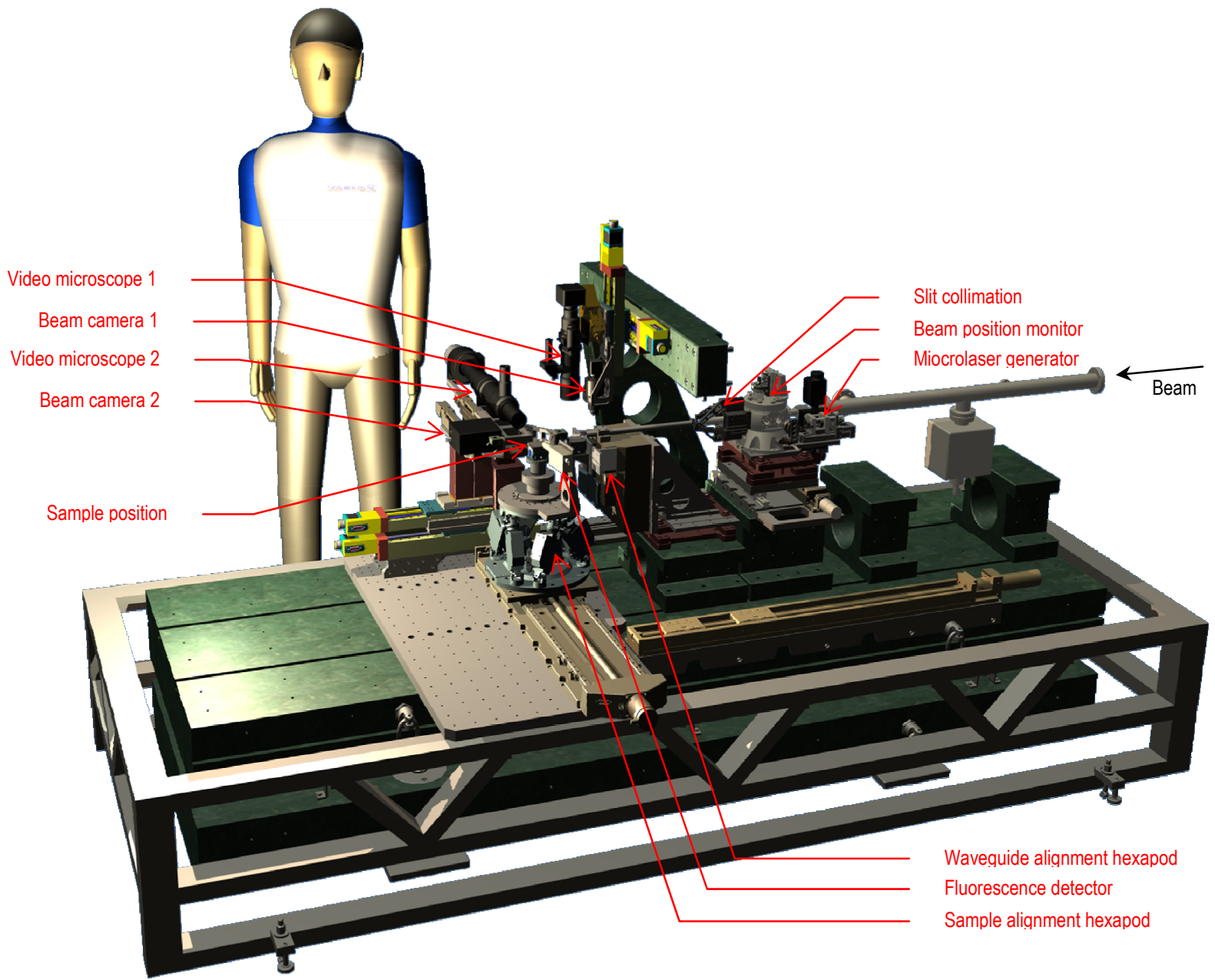

FIGURE 1. Rendered 3D CAD model of the experimental setup to be used at the nanofocus endstation. The person model is to scale. Note the low beam height above ground, traceable by the flight tube on the right side of the model. For visual clarity the front support for the cross bar (where e.g. the video microscope 1 is attached) was removed. 


\section{GENERATION OF THE NANOFOCUS}

The MINAXS beamline is designed to provide a monochromatic or pink X-ray beam within the energy range of $8-23 \mathrm{keV}$. It is therefore beneficial to employ achromatic optical elements for focusing the X-ray onto the desired spot size of $\sim(100 \mathrm{~nm})^{2}$ in the nanofocus endstation. To account for both the large source-sample distance of $\sim 99 \mathrm{~m}$ and the required high demagnification rate, in this operation mode a combination of two different optical devices will be used, as shown schematically in figure 2 .

In a first step the undulator source, with a size of $376 \times 23 \mu \mathrm{m}^{2}(\mathrm{H} \times \mathrm{V})$ and a divergence of $18 \times 11 \mu \mathrm{rad}^{2}(\mathrm{H} \times$ $\mathrm{V}$ ), will be focused by means of a beryllium compound refractive lens (CRL) [3] into a focal spot of $\sim 15 \times 10 \mu \mathrm{m}^{2}$ situated at $\sim 79 \mathrm{~m}$ from the source. By doing this a second, virtual source is generated at this intermediate distance. This virtual source can then be demagnified onto the targeted spot size of $70 \times 110 \mathrm{~nm}^{2}$ by means of a set of elliptically curved mirrors in Kirkpatrick-Baez geometry (KB-mirror) [4] installed beforehand the sample position. This configuration enables to generate the nanofocus in a way that conserves most of the flux provided by the undulator source.

The first option to be realized will be to employ a $2 \mathrm{D}$ waveguide instead or in combination with the aforementioned KB-mirror in order to confine the incident X-ray wave field onto a spot with a size of $\leq(100 \mathrm{~nm})^{2}$ [5]. In this configuration the CRL is used to concentrate as much as possible of the incident flux onto the entrance of the $2 \mathrm{D}$ waveguide, the latter having dimensions in the order of the desired spot size, i.e. also $\leq(100 \mathrm{~nm})^{2}$. To achieve this, the CRL focal length is extended to match the position of the waveguide (shortly before the sample position). In the final step of extension, to further increase the flux being accepted by the waveguide input, the focal spot generated by the KB mirror will be used to couple the X-ray beam into the waveguide. In the waveguide-based configurations, despite the larger divergence of the exiting X-rays (compared to KB-mirror based only focusing) the major advantage of using the waveguide is the high degree of coherence of the wave field exiting the waveguide, which is not achievable with refractive or reflecting optics alone.

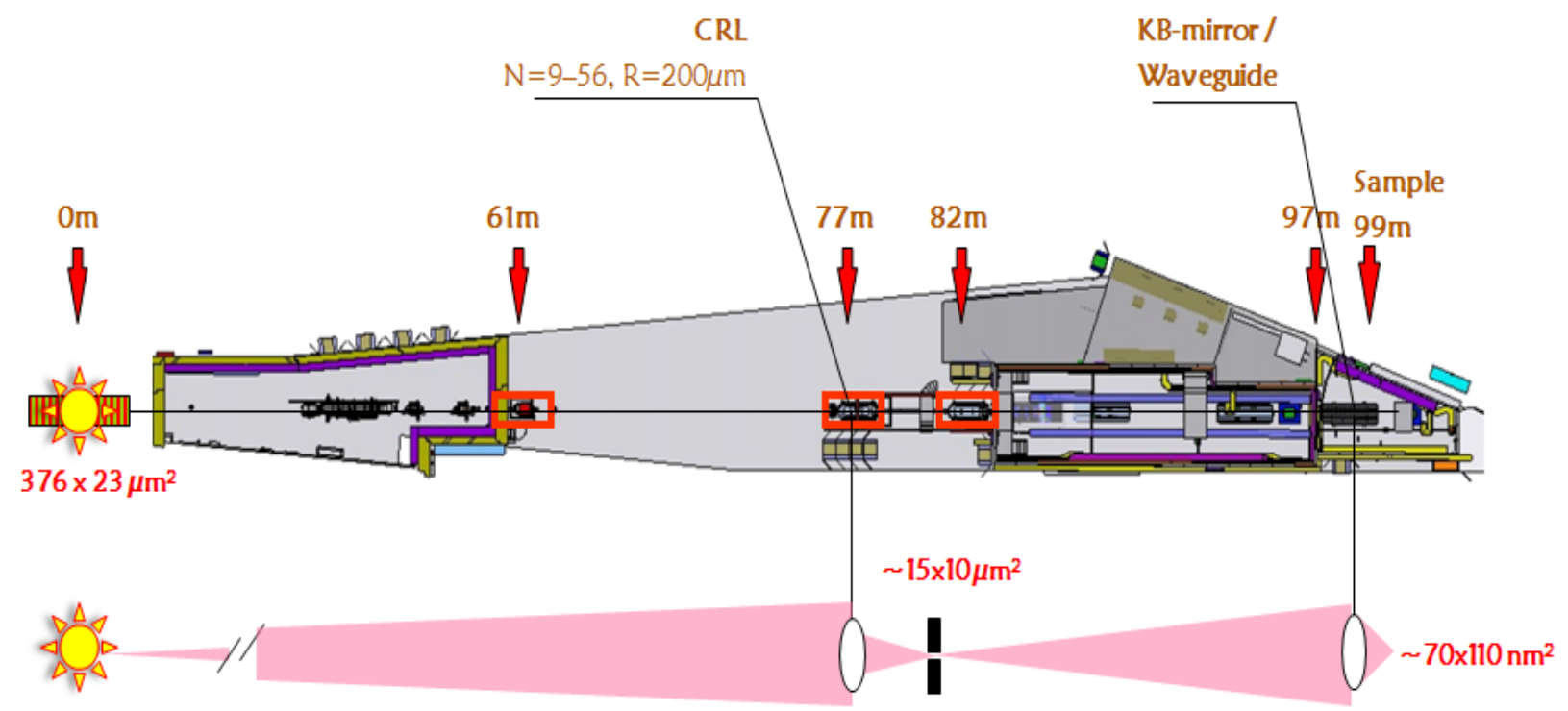

FIGURE 2. Schematic outline of the focusing process to generate the submicron X-ray spot in the nanofocus endstation of MINAXS, exemplarily based on employing a combination of compound refractive lenses (CRL) and a Kirkpatrick Baez (KB) mirror. 


\section{SUMMARY}

The Micro- and Nanofocus X-ray Scattering (MINAXS) beamline of PETRA III is currently in its final state of completion. It is equipped with two endstations, out of which the farthest (nanofocus endstation) is destined to provide a high flux X-ray beam focused to a size of about $(100 \mathrm{~nm})^{2}$ to be used routinely for diffraction experiments. A highly flexible experimental setup in terms of sample alignment and optical element configuration has been engineered and is currently being assembled. Due to the sub-micrometer-sized focus experiments with a superior spatial resolution and a flux sufficiently high to study both biological and synthetic materials will become available at this endstation. Among the potential applications are diffraction methods for the determination of the structure of synthetic materials (e.g. metallurgy, surface science, and semiconductor technology) and biological materials (especially those with multiple levels of structural hierarchy and/or composite materials, such as fibres, cells, etc.). The high potential for new and pathbreaking insight especially for life and materials science as well as nanocomposite and soft matter research is obvious on the basis of the availability of sufficiently high flux density; the latter of course being a key feature of the new synchrotron radiation source PETRA III.

\section{ACKNOWLEDGMENT}

The financial support from the German Federal Ministry of Education and Research (BMBF) within the BMBFVerbundforschung „Erforschung kondensierter Materie an Großgeräten“ (Project BMBF 05KS7FK1) is acknowledged.

\section{REFERENCES}

1. H. Franz, O. Leupold, R. Röhlsberger, S. V. Roth, O. H. Seeck, J. Spengler, J. Strempfer, M. Tischer, J. Viefhaus, E. Weckert, T. Wroblewski, Synchrotron Radiation News 19, 25-29 (2006).

2. S.V. Roth, R. Döhrmann, R. Gehrke, H. Schulte-Schrepping, U. Hahn, H. Franz, R. Röhlsberger, M. Lohmann, H. Graafsma, E. Weckert, HASYLAB Annual Report 2007, pp. 175 - 179.

3. B. Lengeler, C.G. Schroer, M. Kuhlmann, B. Benner, T.F. Günzler, O. Kurapova, F. Zontone, A. Snigirev, I. Snigireva, J. Phys. D 38, A218-A222 (2005)

4. P. Kirkpatrick, A.V. Baez, J. Opt. Soc. Am. 38 (1948) 766-773

5. A. Jarre, C. Fuhse, C. Ollinger, J. Seeger, R. Tucoulu, T. Salditt, Phys.Rev.Lett. 94, 074801 (2005) 